\title{
The Application of Intuitionistic Fuzzy Soft Opeations to Medical Diagnosis
}

\author{
Lishi Zhang,Shengzhe Gao and Hui Gao \\ School of Science,Dalian Ocean University,Dalian, China \\ dwtag@sohu.com
}

Keywords: Soft set; Interval-valued fuzzy soft set; Attribute; Operations of interval-valued fuzzy soft set ; Choice value.

\begin{abstract}
Molodtsov first proposed the soft set theory, which can be utilized as a general mathematical tool for dealing with fuzzy and uncertain information. As a generalization of soft set, intuitionistic fuzzy soft set is another soft set structure, it is a combination of an interval-valued fuzzy set and a soft set. The aim of this paper is to explore the decision making based on interval-valued fuzzy soft sets, the operations are carried out to integrate the medical information and expert information together, the optimcal choice is calculated to choose the disease which the patient most likely to suffer, a numberical example shows that the approach is reasonable..
\end{abstract}

\section{Introduction}

The soft set theory, originally proposed by Molodtsov [2], is a general mathematical tool to deal with uncertainty, imprecision and fuzziness in information system[3]. Soft set theory has been successfully applied in many fields such as functions smoothness, data analysis, forecasting, game theory, theory of measurement and so on. In recent years, soft set theory has drawn considerable attention [4-8]. By integrating soft set theory with other classical mathematical models, a lot of extensions of soft set model have also been made[9-11], fuzzy soft sets, generalized fuzzy soft sets [12], interval-valued fuzzy soft sets, vague soft sets[13], and intuitionistic fuzzy soft sets are all the models of soft set. At the same time, some approaches of decision making to these extended fuzzy soft sets have also been rendered. Level soft sets was first introduced by Feng et al. [14] to present an adjustable approach to fuzzy soft set based decision making. Jiang et al. [15] generalized the adjustable approach to fuzzy soft sets based decision making by transforming the data into a classical soft set, provided an adjustable approach to intuitionistic fuzzy soft sets based decision making.

The rest of this paper is organized as follows. Section 2 briefly recalls some background knowledge.Section 3 gives an illustrative example to verify our methods.

\section{Preliminaries}

In this section, we will briefly recall some basic concepts and notions, background knowledge of intuitionistic fuzzy soft sets is introduced.

Definition 2.1.[11] Let $X$ a set, a fuzzy set $F$ in $X$ is defined as

$$
F=\left\{\left\langle x, \mu_{F}(x)\right\rangle \mid x \in X\right\}
$$

Where $\mu_{F}(x) \in[0,1]$ is the membership of the degree of element $x \in U$.

Definition 2.2.[11] Let $X$ a set, an intuitionistic fuzzy set (IFS) $A$ in $X$ is defined as $A=\left\{\left\langle x, \mu_{A}(x), v_{A}(x)\right\rangle \mid x \in X\right\}$

Where $\mu_{F}(x) \in[0,1]$ and $v_{F}(x) \in[0,1]$ are the degree of membership and nonmembership of an element $x \in U$.respectively,satisfying $0 \leq \mu_{A}(x)+v_{A}(x) \leq 1, \forall x \in X, \pi_{A}(x)=1-\mu_{A}(x)-v_{A}(x)$ is called the intuitionistic fuzzy index of $x \in U$, Each fuzzy set can be represented as the following intuitionistic fuzzy set

$$
A=\left\{\left\langle x, \mu_{A}(x), 1-\mu_{A}(x)\right\rangle \mid x \in X\right\}
$$


For an IFS $A$, the pair $\left(\mu_{A}(x), v_{A}(x)\right)$ is called an intuitionistic fuzzy value $(I F V)$ [16]. For notational convenience, we denote in what follows an IFV by a $\alpha=(\mu, v)$, Where $\mu \in[0,1]$ and $v \in[0,1], \mu+v \leq 1$.

The following operations on IFVs are introduced in $\mathrm{Xu}$ [17].

Definition 2.3.[17] Let $\alpha_{1}=\left(\mu_{1}, v_{1}\right), \alpha_{2}=\left(\mu_{2}, v_{2}\right)$, and $\alpha=(\mu, v)$ be three IFVs, then

(1) $\alpha_{1}+\alpha_{2}=\left(\mu_{1}+\mu_{2}-\mu_{1} \mu_{2}, v_{1} v_{2}\right)$

(2) $\lambda \alpha=\left(1-(1-\mu)^{\lambda}, v^{\lambda}\right),(\lambda>0)$

Where $\mu_{F}(x) \in[0,1]$ and $\nu_{F}(x) \in[0,1]$ are the degree of membership and nonmembership of an element $x \in U$.respectively, satisfying

$$
0 \leq \mu_{A}(x)+v_{A}(x) \leq 1, \forall x \in X, \pi_{A}(x)=1-\mu_{A}(x)-v_{A}(x)
$$

is called the intuitionistic fuzzy index of $x \in U$, each fuzzy set can be represented as the following intuitionistic fuzzy set

$$
A=\left\{\left\langle x, \mu_{A}(x), 1-\mu_{A}(x)\right\rangle \mid x \in X\right\}
$$

For an IFS $A$, the pair $\left(\mu_{A}(x), v_{A}(x)\right)$ is called an intuitionistic fuzzy value. For notational convenience, we denote in what follows an IFV by $\alpha=(\mu, v)$, Where $\mu \in[0,1]$ and $v \in[0,1], \mu+v \leq 1$.

Now we define the matrix and its operations

Definition 2.4.[17] Let $X$ a set, an intuitionistic fuzzy set (IFS) $A, B$ in $X$ is defined as

$$
A=\left\{\left\langle x, \mu_{A}(x), v_{A}(x)\right\rangle \mid x \in X\right\} ; B=\left\{\left\langle x, \mu_{B}(x), v_{B}(x)\right\rangle \mid x \in X\right\}
$$

The intersection of $A$ and $B$ is

$$
A \cap B=\left\{\left\langle x, \min \left\{\mu_{A}(x), \mu_{B}(x)\right\}, \max \left\{v_{A}(x), v_{B}(x)\right\}\right\rangle \mid x \in X\right\}
$$

Definition 2.5 Let $X=\left\{h_{1}, h_{2}, \ldots \ldots, h_{n}\right\}, E=\left\{e_{1}, e_{2}, \ldots \ldots, e_{m}\right\}, X, E$ and are the initial universe and parameters respectively, an intuitionistic fuzzy set is put as follows

$$
X_{n \times m}=\left(a_{i j}\right)_{n \times m} a_{i j}=\left[\mu_{e_{j}}\left(h_{i}\right), v_{e_{j}}\left(h_{i}\right)\right], i=1,2, \cdots, n ; j=1,2, \cdots, m
$$

is called intuitionistic fuzzy soft matrix.

Example 2.1. Let the universe $U=\left\{x_{1}, x_{2}, x_{3}\right\}$ and $E=\left\{e_{1}, e_{2}, e_{3}, e_{4}\right\}$

The intuitionistic fuzzy soft matrix is

$$
X_{3 \times 4}=\left[\begin{array}{llll}
{[0.3,0.7]} & {[0.2,0.4]} & {[0.3,0.5]} & {[0.2,0.5]} \\
{[0.4,0.5]} & {[0.3,0.4]} & {[0.6,0.3]} & {[0.3,0.5]} \\
{[0.2,0.6]} & {[0.5,0.7]} & {[0.3,0.6]} & {[0.3,0.6]}
\end{array}\right]_{3 \times 4}
$$

\section{Definition 2.6 Let}

$$
X_{n \times m}=\left(a_{i j}\right)_{n \times m}, Y_{m \times p}=\left(b_{i j}\right)_{m \times p},
$$

be two intuitionistic fuzzy soft matrixs, where

$$
a_{i j}=\left[\mu_{e_{j}}\left(x_{i}\right), v_{e_{j}}\left(x_{i}\right)\right], i=1,2, \cdots, n ; j=1,2, \cdots, m ; b_{i j}=\left[\mu_{f_{j}}\left(e_{i}\right), v_{f_{j}}\left(e_{i}\right)\right], i=1,2, \cdots, m ; j=1,2, \cdots, p
$$

the composition of $X_{n \times m}, Y_{m \times p}$ is defined as the follows

$$
Z_{n \times p}=X_{n \times m} Y_{m \times p}=\left(z_{i j}\right)_{n \times p} ; z_{i j}=\left[s_{i j}, t_{i j}\right], i=1,2, \cdots, n ; j=1,2, \cdots, p
$$

Where

$$
\begin{aligned}
& s_{i j}=\max _{k}\left\{\min \left\{\mu_{e_{k}}\left(x_{i}\right), \mu_{f_{j}}\left(e_{k}\right)\right\}\right\}, t_{i j}=\min _{k}\left\{\max \left\{v_{e_{k}}\left(x_{i}\right), v_{f_{j}}\left(e_{k}\right)\right\}\right\}, \\
& i=1,2, \cdots, n ; j=1,2, \cdots, p ; k=1,2, \cdots, m
\end{aligned}
$$

Example 2.2. Let the universe

$$
U=\left\{x_{1}, x_{2}\right\} \text { and } E=\left\{e_{1}, e_{2}, e_{3}\right\} ; U=\left\{e_{1}, e_{2}, e_{3}\right\} \text { and } F=\left\{f_{1}, f_{2}, f_{3}, f_{4}\right\}
$$

The intuitionistic fuzzy soft matrix is

$$
X_{2 \times 3}=\left[\begin{array}{lll}
{[0.2,0.6]} & {[0.3,0.5]} & {[0.4,0.6]} \\
{[0.3,0.6]} & {[0.6,0.2]} & {[0.3,0.2]}
\end{array}\right]_{2 \times 3} ;_{Y_{3 \times 4}}=\left[\begin{array}{llll}
{[0.3,0.7]} & {[0.2,0.4]} & {[0.3,0.5]} & {[0.2,0.5]} \\
{[0.4,0.5]} & {[0.3,0.4]} & {[0.6,0.3]} & {[0.3,0.5]} \\
{[0.2,0.6]} & {[0.5,0.4]} & {[0.3,0.6]} & {[0.6,0.3]}
\end{array}\right]_{3 \times 4}
$$




$$
Z_{2 \times 4}=X_{2 \times 3} \circ Y_{3 \times 4}=\left[\begin{array}{llll}
{[0.3,0.5]} & {[0.4,0.5]} & {[0.3,0.5]} & {[0.4,0.5]} \\
{[0.4,0.5]} & {[0.3,0.4]} & {[0.6,0.3]} & {[0.3,0.3]}
\end{array}\right]_{2 \times 4}
$$

Theorem 2.1[17] The composition of two intuitionistic fuzzy soft matrix is also a intuitionistic fuzzy soft matrix.

Definition 2.7[17] Let $\alpha=(\mu, v)$ be an IFV, then $s(\alpha)=\mu-v$ is called a score of $\alpha$. and s is called the score function, where $s(\alpha) \in[-1,1]$.

Later on, Hong and Choi defined the accuracy function of an IFV as:

Let $\alpha=(\mu, v)$ be an IFV, then $h(\alpha)=\mu+v$ is called an accuracy of a, and $h$ is called the accuracy function, where $s(\alpha) \in[0,1]$. Based on the score function and accuracy function, Xu [17] proposes the following method to compare two IFVs.

Definition 2.8[17] Let $\alpha_{1}=\left(\mu_{1}, v_{1}\right), \alpha_{2}=\left(\mu_{2}, v_{2}\right)$ be two IFVs, If $s\left(\alpha_{1}\right)<s\left(\alpha_{2}\right)$, then $\alpha_{1}$ is smaller than $\alpha_{2}$, denoted by $\alpha_{1}<\alpha_{2}$,

If $s\left(\alpha_{1}\right)=s\left(\alpha_{2}\right)$, and

(1) if $h\left(\alpha_{1}\right)=h\left(\alpha_{2}\right)$, then $\alpha_{1}$ and $\alpha_{2}$ represent the same information denoted by $\alpha_{1}=\alpha_{2}$.

(2) if $h\left(\alpha_{1}\right)<h\left(\alpha_{2}\right)$, then $\alpha_{1}$ is smaller than $\alpha_{2}$, denoted by $\alpha_{1}<\alpha_{2}$.

Definition 2.9[17] Let $\alpha_{i}=\left(\mu_{i}, v_{i}\right),(\mathrm{i}=1,2, \cdots, n)$ be a collection of IFNs, and let IFWA: $\theta^{n} \rightarrow \theta$. and

$$
I F W A_{\omega}\left(\alpha_{1}, \alpha_{2}, \cdots, \alpha_{n}\right)=\omega_{1} \alpha_{1} \oplus \omega_{2} \alpha_{2} \oplus \cdots \oplus \omega_{n} \alpha_{n}(i=1,2, \cdots, n)
$$

is called an intuitionistic fuzzy weighted averaging operator, where $\omega=\left(\omega_{1}, \omega_{2}, \cdots, \omega_{n}\right)^{T}$ is the weight vector of $\alpha_{i}(i=1,2, \cdots, n)$, with $\omega_{i} \in[0,1]$ and $\sum_{i=1}^{n} \omega_{i}=1$.

Definition 2.10[17] Let $\alpha_{1}=\left(\mu_{1}, v_{1}\right), \alpha_{2}=\left(\mu_{2}, v_{2}\right)$ be two IFVs, the distance between $\alpha_{1}$ and $\alpha_{2}$ is

$$
d\left(\alpha_{1}, \alpha_{2}\right)=\frac{\left|\mu_{1}-\mu_{2}\right|+\left|v_{1}-v_{2}\right|}{2}
$$

Definition 2.11[17] Let $X=\left\{x_{1}, x_{2}, \cdots, x_{n}\right\}$

$$
A=\left\{\left\langle x, \mu_{A}(x), v_{A}(x)\right\rangle \mid x \in X\right\} ; B=\left\{\left\langle x, \mu_{B}(x), v_{B}(x)\right\rangle \mid x \in X\right\}
$$

be two IFVs, the distance between $A$ and $B$ is

$$
d(A, B)=\frac{1}{2 n} \sum_{i=1}^{n}\left[\left|\mu_{A}\left(x_{i}\right)-\mu_{B}\left(x_{i}\right)\right|+\left|v_{A}\left(x_{i}\right)-v_{B}\left(x_{i}\right)\right|\right]
$$

\section{Case Study}

Let there be four patients Paul, Jadu, Kundu, and Rohit in hospital at Calcutta. Their symptoms are temperature headache stomach pain, cough and chest-pain. Clearly, the Set of patients

$P=\{$ Paul, Jadu, Kundu, Rohit $\}=\left\{h_{1}, h_{2}, h_{3}, h_{4}\right\}$

The set of symptoms

$S=\{$ temperature, headache, stomach pain, cough ,chest-pain $\}=\left\{e_{1}, e_{2}, e_{3}, e_{4}, e_{5}\right\}$,

The set of disease

$D=\{$ Viral Fever, Malaria, Typhoid,Stomach problem,Heart problem $\}=\left\{d_{1}, d_{2}, d_{3}, d_{4}, d_{5}\right\}$,

The intuitionistic fuzzy soft sets of patients and symptoms are represented as

$$
\begin{aligned}
& F\left(e_{1}\right)=\left\{\left\langle h_{1},[0.8,0.1]\right\rangle,\left\langle h_{2},[0,0.8]\right\rangle,\left\langle h_{3},[0.8,0.1]\right\rangle,\left\langle h_{4},[0.6,01]\right\rangle\right\} . \\
& F\left(e_{2}\right)=\left\{\left\langle h_{1},[0.6,0.1]\right\rangle,\left\langle h_{2},[0.4,0.4]\right\rangle,\left\langle h_{3},[0.8,0.1]\right\rangle,\left\langle h_{4},[0.5,0.4]\right\rangle\right\} . \\
& F\left(e_{3}\right)=\left\{\left\langle h_{1},[0.2,0.8]\right\rangle,\left\langle h_{2},[0.6,0.1]\right\rangle,\left\langle h_{3},[0,0.6]\right\rangle,\left\langle h_{4},[0.3,0.4]\right\rangle\right\} . \\
& F\left(e_{4}\right)=\left\{\left\langle h_{1},[0.6,0.1]\right\rangle,\left\langle h_{2},[0.1,0.7]\right\rangle,\left\langle h_{3},[0.2,0.7]\right\rangle,\left\langle h_{4},[0.7,0.2]\right\rangle\right\} . \\
& F\left(e_{5}\right)=\left\{\left\langle h_{1},[0.1,0.6]\right\rangle,\left\langle h_{2},[0.1,0.8]\right\rangle,\left\langle h_{3},[0,0.5]\right\rangle,\left\langle h_{4},[0.3,0.4]\right\rangle\right\} .
\end{aligned}
$$

The intuitionistic fuzzy soft sets of symptoms with respect to disease are represented as 


$$
\begin{aligned}
& G\left(d_{1}\right)=\left\{\left\langle e_{1},[0.4,0]\right\rangle,\left\langle e_{2},[0.3,0.5]\right\rangle,\left\langle e_{3},[0.1,0.7]\right\rangle,\left\langle e_{4},[0.4,0.3]\right\rangle,\left\langle e_{5},[0.1,0.7]\right\rangle\right\} . \\
& G\left(d_{2}\right)=\left\{\left\langle e_{1},[0.7,0]\right\rangle,\left\langle e_{2},[0.2,0.6]\right\rangle,\left\langle e_{3},[0,0.9]\right\rangle,\left\langle e_{4},[0.7,0]\right\rangle,\left\langle e_{5},[0.1,0.8]\right\rangle\right\} . \\
& G\left(d_{3}\right)=\left\{\left\langle e_{1},[0.3,0.3]\right\rangle,\left\langle e_{2},[0.6,0.1]\right\rangle,\left\langle e_{3},[0.2,0.7]\right\rangle,\left\langle e_{4},[0.2,0.6]\right\rangle,\left\langle e_{5},[0.1,0.9]\right\rangle\right\} . \\
& G\left(d_{4}\right)=\left\{\left\langle e_{1},[0.1,0.7]\right\rangle,\left\langle e_{2},[0.2,0.4]\right\rangle,\left\langle e_{3},[0.8,0]\right\rangle,\left\langle e_{4},[0.2,0.7]\right\rangle,\left\langle e_{5},[0.2,0.7]\right\rangle\right\} . \\
& G\left(d_{5}\right)=\left\{\left\langle e_{1},[0.1,0.8]\right\rangle,\left\langle e_{2},[0,0.8]\right\rangle,\left\langle e_{3},[0.2,0.8]\right\rangle,\left\langle e_{4},[0.2,0.8]\right\rangle,\left\langle e_{5},[0.8,0.1]\right\rangle\right\} .
\end{aligned}
$$

The matrix presentation is

$$
\begin{gathered}
X_{4 \times 5}=\left[\begin{array}{ccccc}
{[0.8,0.1]} & {[0.6,0.1]} & {[0.2,0.8]} & {[0.6,0.1]} & {[0.1,0.6]} \\
{[0,0.8]} & {[0.4,0.4]} & {[0.6,0.1]} & {[0.1,0.7]} & {[0.1,0.8]} \\
{[0.8,0.1]} & {[0.8,0.1]} & {[0,0.6]} & {[0.2,0.7]} & {[0,0.5]} \\
{[0.6,0.1]} & {[0.5,0.4]} & {[0.3,0.4]} & {[0.7,0.2]} & {[0.3,0.4]}
\end{array}\right]_{4 \times 5} \\
Y_{5 \times 5}=\left[\begin{array}{ccccc}
{[0.4,0.1]} & {[0.7,0]} & {[0.3,0.3]} & {[0.1,0.7]} & {[0.8,0.1]} \\
{[0.3,0.5]} & {[0.2,0.6]} & {[0.6,0.1]} & {[0.2,0.4]} & {[0,0.8]} \\
{[0.1,0.7]} & {[0,0.9]} & {[0.2,0.7]} & {[0.8,0]} & {[0.2,0.8]} \\
{[0.4,0.3]} & {[0.7,0]} & {[0.2,0.6]} & {[0.2,0.7]} & {[0.2,0.8]} \\
{[0.1,0.7]} & {[0.1,0.8]} & {[0.1,0.9]} & {[0.2,0.7]} & {[0.8,0.1]}
\end{array}\right]_{5 \times 5}
\end{gathered}
$$

The composition of the two matrix is

$$
Z_{4 \times 5}=\left[\begin{array}{lllll}
{[0.4,0.1]} & {[0.7,0.1]} & {[0.6,0.1]} & {[0.2,0.4]} & {[0.2,0.6]} \\
{[0.3,0.5]} & {[0.2,0.6]} & {[0.4,0.4]} & {[0.6,0.1]} & {[0.1,0.7]} \\
{[0.4,0.1]} & {[0.7,0.1]} & {[0.6,0.1]} & {[0.2,0.4]} & {[0.2,0.5]} \\
{[0.4,0.1]} & {[0.7,0.1]} & {[0.5,0.3]} & {[0.3,0.4]} & {[0.3,0.4]}
\end{array}\right]_{4 \times 5}
$$

With the score function, we get that

\begin{tabular}{|l|l|c|l|l|l|}
\hline$U$ & Viral Fever & Malaria & Typhoid & Stomach & Heart problem \\
\hline Paul & 0.3 & 0.6 & 0.5 & -0.2 & -0.4 \\
\hline Jadu & -0.2 & -0.4 & 0 & 0.5 & -0.6 \\
\hline Kundu & 0.3 & 0.6 & 0.5 & -0.2 & -0.3 \\
\hline Rohit & 0.3 & 0.6 & 0.2 & -0.1 & -0.1 \\
\hline
\end{tabular}

From the above table, we see that Paul, Kundu, Rohit suffer from the disease Malaria, while Jadu suffers from the disease stomach. The result is in agreement with [18],Let us observe another example Let us consider the following pattern recognition problem as discussed in [19]. given five known patterns, which corresponds to five decision alternatives $d_{1}, d_{2}, d_{3}, d_{4}, d_{5}$ respectively. The patterns are denoted by the following IvIF

$$
\begin{aligned}
X & =\left\{x_{1}, x_{2}, x_{3}, x_{4}\right\} \\
R_{1} & =\left\{\left\langle h_{1},[0.4,0.4]\right\rangle,\left\langle h_{2},[0.4,0.4]\right\rangle,\left\langle h_{3},[0.3,0.5]\right\rangle,\left\langle h_{4},[0.5,0.3]\right\rangle\right\} \\
R_{2} & =\left\{\left\langle h_{1},[0.5,0.3]\right\rangle,\left\langle h_{2},[0.6,0.3]\right\rangle,\left\langle h_{3},[0.5,0.4]\right\rangle,\left\langle h_{4},[0.4,0.2]\right\rangle\right\} \\
R_{3} & =\left\{\left\langle h_{1},[0.3,0.4]\right\rangle,\left\langle h_{2},[0.1,0.6]\right\rangle,\left\langle h_{3},[0.2,0.5]\right\rangle,\left\langle h_{4},[0.2,0.6]\right\rangle\right\} \\
R_{4} & =\left\{\left\langle h_{1},[0.2,0.4]\right\rangle,\left\langle h_{2},[0.4,0.2]\right\rangle,\left\langle h_{3},[0.4,0.5]\right\rangle,\left\langle h_{4},[0.5,0.2]\right\rangle\right\} \\
R_{5} & =\left\{\left\langle h_{1},[0.3,0.3]\right\rangle,\left\langle h_{2},[0.7,0.2]\right\rangle,\left\langle h_{3},[0.5,0.4]\right\rangle,\left\langle h_{4},[0.6,0.2]\right\rangle\right\}
\end{aligned}
$$

Given an unknown sample

$$
r=\left\{\left\langle h_{1},[0.5,0.3]\right\rangle,\left\langle h_{2},[0.7,0.2]\right\rangle,\left\langle h_{3},[0.5,0.4]\right\rangle,\left\langle h_{4},[0.6,0.2]\right\rangle\right\}
$$

Our aim is to classify pattern $r$ to one of the decision alternatives $d_{1}, d_{2}, d_{3}, d_{4}$, and $d_{5}$. That is, we will find which one of the five decisions is mostly close to the unknown sample $r$. 


$$
\begin{aligned}
& I F W A_{\omega}\left(R_{1} \cap r\right)=[0.3519,0.3936], I F W A_{\omega}\left(R_{2} \cap r\right)=[0.5271,0.2913], I F W A_{\omega}\left(R_{3} \cap r\right)=[0.2031,0.5180] \\
& I F W A_{\omega}\left(R_{4} \cap r\right)=[0.3553,0.2991], I F W A_{\omega}\left(R_{5} \cap r\right)=[0.5213,0.2632] \\
& S\left(R_{1} \cap r\right)=-0.0417 ; S\left(R_{2} \cap r\right)=0.2358 ; S\left(R_{3} \cap r\right)=-0.3149 ; S\left(R_{4} \cap r\right)=0.0562 ; S\left(R_{5} \cap r\right)=0.2581 ;
\end{aligned}
$$

The order of scores are listed as follows

$$
S\left(R_{5} \cap r\right)>S\left(R_{2} \cap r\right)>S\left(R_{4} \cap r\right)>S\left(R_{1} \cap r\right)>S\left(R_{3} \cap r\right)
$$

It means that the decision alternative $d_{5}$ is the optimal alternative which is the closest alterative to positive ideal solution. This result is in agreement with the one obtained in [19].

\section{References}

[1] Y. Celik, S. Yamak. Fuzzy soft set theory applied to medical diagnosis using fuzzy arithmetic operations, Journal of Inequalities and Applications,82(2013)1-9.

[2] D. Molodtsov. Soft set theory-first results, Comput. Math. Appl, 37(1999)19-31.

[3] L.A. Zadeh. Fuzzy Sets, Inf. Control, 8(1965)338-353.

[4] M.I. Ali, F. Feng and S. Liu. On some new operations in soft set theory, Comput. Math. Appl. 57(2009)1547-1553.

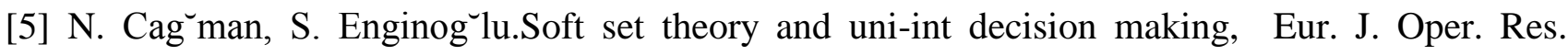
207(2010)848-855.

[6] K. Gong, P.P. Wang and Z. Xiao.Bijective soft set decision system based parameters reduction under fuzzy environments, Appl. Math. Model. 37(2013)4474-4485.

[7] Z. Kong, L. Gao and L.F. Wang. The normal parameter of soft sets and its algorithm,Comput. Math. Appl. 56(2008)3029-3037.

[8]Y. Zou, Z. Xiao.Data analysis approaches of soft sets under incomplete information, Knowl.-Based Syst, 21(2008)941-945.

[9] T.Q. Deng, X.F. Wang. An object-parameter approach to predicting unknown data in incomplete fuzzy soft sets, Appl. Math. Model,37(2013)4139-4146.

[10] T.M. Basu, N.K. Mahapatra and S.K. Mondal, A balanced solution of a fuzzy soft set based decision making problem in medical science, Appl. Soft Comput, 12(2012)3260-3275.

[11] Z.M. Zhang. A rough set approach to intuitionistic fuzzy soft set based decision making, Appl. Math. Model, 36(2012)4605-4633.

[12] P.Majumdar,S.K.Samanta.Generalised fuzzy soft sets,Comput.Math.Appl, 59(2010)1425-1432.

[13]W.Xu,J.Ma and S.Y.Wang.Vague soft sets and their properties, Comput.Math.Appl,59(2010)787-794.

[14] F. Feng, Y.B. Jun and X.Y. Liu. An adjustable approach to fuzzy soft set based decision making, J. Comput. Appl. Math, 234(2010)10-20.

[15] Y. Jiang, Y. Tang and Q. Chen.An adjustable approach to intuitionistic fuzzy soft sets based decision making, Appl. Math. Model, 35(2011)824-836.

[16] Y. Jiang, Y. Tang and Q. Chen.Interval-valued intuitionistic fuzzy soft sets and their properties, Comput. Math. Appl,60(2010)906-918. 
[17]Z.S.Xu. A deviation-based approach to Interval-valued intuitionistic fuzzy multiple attribute group decision making, Group Decision and Negotiation,19(2010)57-76.

[18]De.Sk,Biswas,R,Roy.An application of intuitionistic fuzzy sets in medical diagnosis, Fuzzy Sets Syst ,117(2001)209-213.

[19]Q.S.Zhang,S.Y.Jiang and B.G.Jia.Some information measures for Interval-valued intuitionistic fuzzy sets,Information Science , 180(2010)5130-5145. 